\title{
Expression of FMRP by Neurosphere Derived Cells Following Transplantation in the Fragile X Mouse Model
}

\author{
Laura K. K. Pacey ${ }^{1}$ and Laurie C. Doering ${ }^{2 *}$
}

${ }^{1}$ Leslie Dan Faculty of Pharmacy, University of Toronto, 144 College Street, Toronto, Ontario, Canada M5S 3M2

${ }^{2}$ Department of Pathology and Molecular Medicine, McMaster University, 1280 Main Street West, Hamilton, Ontario, Canada L8S $4 K 1$

\begin{abstract}
Fragile X Syndrome, characterized by intellectual impairment and learning disabilities is caused by a trinucleotide repeat expansion in the $\mathrm{x}$-linked FMR1 gene leading to a complete lack of Fragile X Mental Retardation Protein (FMRP). Neural stem/progenitor cells (NSCs) cultured as floating aggregates of cells commonly referred to as neurospheres can survive, differentiate and integrate into the host environment when transplanted to the rodent CNS. Neurospheres were established from early postnatal wild-type mice and labeled with the cytoplasmic dye CFDA-SE and then stereotaxically injected as cell suspensions into the hippocampus of young adult (3-6 week old) FMR1 knockout mice. Immunocytochemical analysis of the brains revealed the presence of FMRP-expressing cells up to two weeks after transplantation. Some transplanted cells migrated within the host hippocampus and formed dendrite-like processes. A small subpopulation of the transplanted cells expressed markers of neuronal or glial phenotypes, although many cells did not stain for any of the markers that we probed. These experiments constitute an attempt to restore FMRP-expression in the brains of FMR1 knockout mice using cell suspension transplants of stem and progenitor cells.
\end{abstract}

Keywords: Fragile X syndrome: Mouse model; Neurosphere; Transplant; FMRP

\section{Introduction}

Cell replacement studies have become an important tool for studying many aspects of neurobiology. Transplantation studies have fundamental aims in neuroscience: to explore the basic principles important in neurodevelopment or to treat a specific neurological disorder. The discovery of neural stem cells in the adult brain has provided new alternative cell populations for transplantation and regenerative studies. When transplanted to models of the normal and diseased CNS, neural stem and progenitor cells can produce neurons, astrocytes and oligodendrocytes [1,2]. With a wide differentiation potential, these cells are attractive cell candidates for transplantation studies in the CNS.

In the case of Fragile X Syndrome (FXS), one of the most common inherited forms of mental retardation, individuals lack functional Fragile X Mental Retardation Protein (FMRP). FMRP, the protein product of the FMR1 gene, associates with specific proteins, mRNA and ribosomes to form messenger ribonucleoprotein (mRNP) complexes [3] which are involved in the transport and translation of mRNA. Within the brain, the highest concentrations of FMRP are found in the hippocampus, cerebellum and cerebral cortex $[4,5]$.

In this study we sought to determine if stem/progenitor cells could express FMRP following transplantation to the hippocampus of the Fragile X mouse model [6]. The morphological characteristics and fate of pre-labeled cells were studied with immunofluorescent techniques after stereotaxic transplantation to the brains of FMRP knockout mice.

\section{Materials and Methods}

All experiments with the Fragile X (FVB.129P2(B6)-Fmr $1^{\text {tmlCgr }}$ ) mice were carried out in accordance with the guidelines set out by the Canadian Council on Animal Care and they were approved by the Animal Research Ethics Board of McMaster University.

\section{Neurospheres}

Wildtype and knockout (FVB.129P2 (B6)-Fmr1 ${ }^{\text {tmlCgr }}$ ) mouse pups (2-7 days old) were euthanised and the brains processed for the neurosphere assay [7]. Briefly, the brain, excluding the cerebellum, was cut into small pieces, transferred to $1.0 \mathrm{~mL}$ of dissecting solution containing enzymes in concentrations listed by Pacey et al. [7] and incubated at $35^{\circ} \mathrm{C}$ in a shaking waterbath for 45 minutes. The mixture was subsequently transferred to a $1.0 \mathrm{mg} / \mathrm{mL}$ solution of trypsin inhibitor (Roche) in serum free medium and incubated at $35^{\circ} \mathrm{C}$ for 10 minutes. The mixture was reconstituted in $12.0 \mathrm{~mL}$ of serum free medium consisting of DMEM-F12 (Invitrogen), glucose, HEPES buffer (Sigma), putrescine (Sigma), progesterone (Sigma), insulin-transferrin-sodium-selenite (Roche), B27 growth supplement (Invitrogen), epidermal growth factor (Sigma), basic fibroblast growth factor (Sigma) and heparin (Sigma). Cells were plated in 24 well dishes (Falcon), incubated in 95\% air, 5\% $\mathrm{CO}_{2}$ and passaged every 4-7 days.

\section{In vitro differentiation and characterization}

Neurospheres in culture were transferred to fresh serum-free medium, containing half the normal concentrations of EGF, FGF-2 and heparin in 24 well plates containing glass coverslips coated with $0.1 \mathrm{mg} / \mathrm{mL}$ poly-L-lysine (Sigma) and $10 \mu \mathrm{g} / \mathrm{mL}$ laminin (Sigma). After 7 days of incubation, the coverslips with the differentiated cells were

*Corresponding author: Laurie C. Doering, Department of Pathology and Molecular Medicine, McMaster University, 1280 Main Street West, Hamilton, Ontario, Canada L8S 4K1, E-mail: doering@mcmaster.ca

Received December 16, 2011; Accepted December 26, 2011; Published January 02, 2012

Citation: Pacey LKK, Doering LC (2012) Expression of FMRP by Neurosphere Derived Cells Following Transplantation in the Fragile X Mouse Model. J Stem Cell Res Ther S12:001. doi:10.4172/2157-7633.S12-001

Copyright: @ 2012 Pacey LKK, et al. This is an open-access article distributed under the terms of the Creative Commons Attribution License, which permits unrestricted use, distribution, and reproduction in any medium, provided the original author and source are credited. 
washed in $0.01 \mathrm{M}$ phosphate buffered saline (PBS), and then fixed in $4 \%$ paraformaldehyde (PFA) for 15 minutes at room temperature. Coverslips were washed twice with sterile PBS and incubated overnight at $4^{\circ} \mathrm{C}$ with the primary antibodies: FMRP $2 \mathrm{~F} 5-1$ (gift from Dr. J. Fallon), GFAP (DAKO), ßIII-tubulin (Chemicon), NF68 (Sigma), nestin (PharMingen), PGP 9.5 (Chemicon), NG2 (Chemicon), vimentin (Chemicon). Coverslips were washed three times with PBS and incubated with the species specific secondary antibody for 3 hours at room temperature. Controls were treated with only the secondary antibody. Slides were mounted using Vectashield mounting medium with DAPI (Vector Labs).

\section{Preparing neurospheres for transplantation}

Neurospheres in culture for 2 to 7 days were removed from the wells and centrifuged for 5 minutes at $2000 \mathrm{rpm}$. Cells were resuspended in $2.0 \mathrm{~mL}$ TrypLE ${ }^{\bullet}$ express (Invitrogen) and incubated for 30 minutes in a $37^{\circ} \mathrm{C}$ shaking waterbath. Dissociated cells were labeled with the Vybrant $^{\mathrm{TM}}$ CFDA SE Cell Tracer Kit (Molecular Probes) as per the manufacturer's instructions. Briefly, dissociated cells were resuspended in $1.0 \mathrm{~mL}$ sterile PBS containing $5.0 \mu \mathrm{L}$ of $10 \mu \mathrm{M}$ CFDA solution and incubated at $37^{\circ} \mathrm{C}, 95 \%$ air, $5 \% \mathrm{CO}_{2}$ for 15 minutes. The CFDA solution was removed and the cells were resuspended in $1.0 \mathrm{~mL}$ serum free media and incubated at $37^{\circ} \mathrm{C}, 95 \%$ air, $5 \% \mathrm{CO}_{2}$ for 30 minutes. Cells were washed twice and resuspended in $15-30 \mu \mathrm{L}$ sterile lactated Ringers solution. Cell viability counts were obtained using Trypan blue exclusion using a 1:4 ratio of trypan blue to the cell suspension. The cell suspension was placed on ice in preparation for transplantation.

\section{Transplantation procedures}

Young adult (3-6 weeks) and adult (6 months) male FVB.129P2 (B6)-Fmr1 ${ }^{\text {tmlCgr }}$ mice were anesthetized with an intraperitoneal injection $(0.1 \mathrm{~mL} / 10 \mathrm{~g})$ of a mixture of $15 \%$ ketamine and $5 \%$ xylazine and placed in a Kopf stereotaxic apparatus.

A $10 \mathrm{~mL}$ Hamilton syringe was used to inject a total of $3.0 \mu \mathrm{L}$ of labeled cell suspension (0.1-6 x $10^{4}$ viable cells) into the right hippocampus (AP $-2.54 \mathrm{~mm}$, ML $2.0 \mathrm{~mm}$, DV $1.5 \mathrm{~mm}$ from Bregma). The needle was inserted at the specified coordinates and $1.0 \mu \mathrm{L}$ of cell suspension injected at a rate of $0.8 \mu \mathrm{L} / \mathrm{min}$. The needle was moved up approximately $0.3 \mathrm{~mm}$ (twice), with $1 \mu \mathrm{L}$ of cells injected each time. In total, $3.0 \mu \mathrm{L}$ of the cell suspension was injected over approximately 1.0 $\mathrm{mm}$. The needle was left in place for one minute after the infusion. Mice were given buprenorphine (subcutaneous) and xylocaine (topical) before surgery as preventative analgesics. For controls, CFDA-SE labeled cells were killed by placing the cells in boiling water for 3 minutes, followed by flash freezing on ice. Cell viability was assessed by trypan blue exclusion. The solution of dead labeled cells $(3.0 \mu \mathrm{L})$ was injected as described above. Negative controls were injected with $3.0 \mu \mathrm{L}$ of sterile lactated Ringers solution.

\section{Analysis of brain sections from transplanted animals}

One, seven, fourteen and twenty-one days after transplantation, mice were deeply anaesthetized with an intra-peritoneal injection of $3.0 \%$ chloral hydrate. Mice were perfused through the heart with $0.01 \mathrm{M}$ PBS followed by $4.0 \%$ paraformaldehyde (PFA) and the brain removed and immersed in $4 \%$ PFA overnight at $4^{\circ} \mathrm{C}$. The following morning, the brains were equilibrated in $30 \%$ sucrose in $\mathrm{PBS}$ at $4^{\circ} \mathrm{C}$ overnight. Frozen cryostat sections, 18 to $20 \mu \mathrm{m}$ thick, were immersed in cold $0.01 \mathrm{M}$ PBS then transferred to glass test tubes and allowed to equilibrate to room temperature. The sections were treated with $0.8 \% \mathrm{Na}$ borohydride (Sigma) in PBS for 10 minutes, washed with PBS and incubated for 45 minutes in $0.01 \mathrm{M} \mathrm{Na}$ citrate buffer, $\mathrm{pH} 6.0$, at $75^{\circ} \mathrm{C}$ in a shaking water bath. Once cooled, sections were rinsed with PBS, permeabilized with $0.1 \%$ Triton X-100 in PBS for 5 minutes and blocked using the Mouse on Mouse (MOM) Immunodetection Kit (Vector Labs). Primary antibodies were applied overnight at $4^{\circ} \mathrm{C}$ and the secondary antibodies applied for 1 hour at room temperature. When applicable, cells were washed with PBS and Avidin DCS was applied for 10 minutes at room temperature. Slides were mounted using Vectashield mounting medium with DAPI (Vector Labs).

\section{Results}

\section{Wildtype neurospheres produce neurons and glia in vitro}

After 7 days in vitro, differentiated neurospheres were immunopositive for neurons ( $\beta$ III-tubulin; NF68) and astrocytes (GFAP; S100 $\beta$ ). Analysis of an average of approximately 400 cells from each of three separate experiments indicated that $55.0 \pm 1.1 \%$ (mean + SEM) of differentiated cells expressed GFAP and showed an astrocytic morphology and $10.5 \pm 3.6 \%$ of cells expressed $\beta$ III-tubulin and showed a typical neuronal morphology (Figure $1 \mathrm{~A}$ and B). In a separate set of three experiments, where an average of approximately 450 cells were counted $4.3 \pm 0.8 \%$ of cells expressed NG2 and demonstrated a morphology characteristic of oligodendrocyte precursor cells (Figure $1 C)$.

Many cells derived from differentiated neurospheres co-expressed glial and neuronal markers, suggesting these cells were not committed to a particular fate. When three separate experiments were analyzed, $53.5 \pm 0.5 \%$ of differentiated cells co-expressed $\beta I I I$-tubulin and GFAP in vitro (Figure 1D). For individual cells co-expressing GFAP and $\beta I I I-$
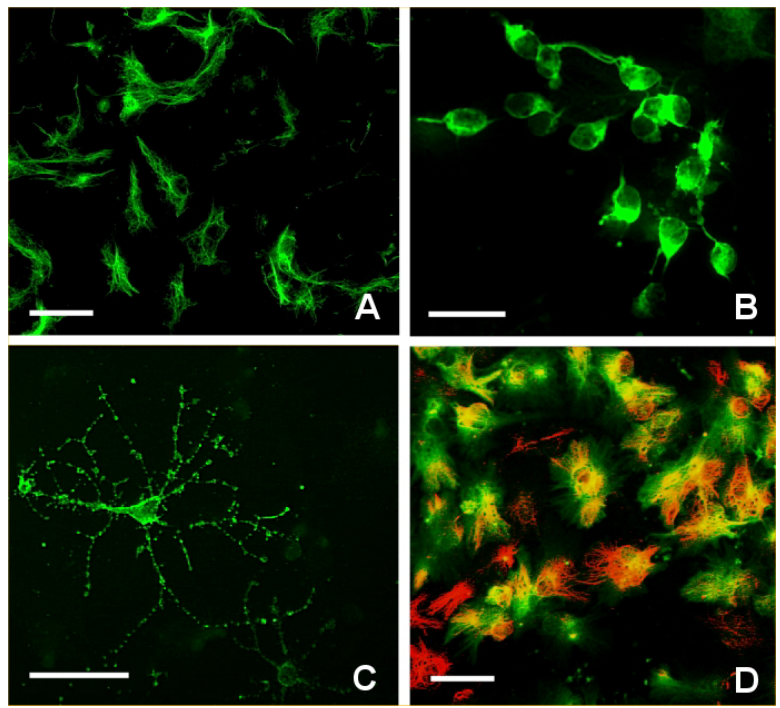

Figure 1: In vitro differentiation of neurosphere derived cells. A) Expression of GFAP defines astrocytes with typical flattened cell morphology. B) Immunocytochemical staining of cells with $\beta$ III-tubulin. C) Staining of a cell with the NG2 antibody outlines the cell processes and cell body of an oligodendrocyte. D) Numerous cells co-expressed GFAP (green) and BIII-tubulin (red) reflecting an uncommitted cell lineage. Indirect immunofluorescence on cells in 7 day old cultures. Scale bar $=25 \mu \mathrm{m}$. 
tubulin, the level of expression varied suggesting that these cells may be upregulating one antigen and downregulating the other as they move towards a committed fate. In addition, the vast majority of these cells had an astrocyte-like morphology, with very few double labeled cells showing a small, round, bipolar morphology similar to neuronal cells.

\section{Wildtype neurospheres produce FMRP expressing cells}

After 7 days in vitro, wildtype neurospheres produced cells that expressed FMRP (Figure 2). Many FMRP-positive cells co-expressed both neuronal ( $\beta$ III-tubulin; PGP9.5; NF68; Figure 2A and B) and/or glial markers (GFAP; NG2; Figure 2C and D). Some cells exhibiting an uncommitted fate, such as those expressing vimentin and those coexpressing GFAP and BIII-tubulin were also found to express FMRP (not shown). Previous quantification showed that approximately $60 \%$ of differentiated cells expressed FMRP after 7 days [8]. No FMRP immunoreactivity was observed after differentiation of the neurospheres derived from the FMR1 knockout mice.

\section{CFDA labeled cells}

In order to validate the appropriate use of CFDA as a label for our neurosphere-derived cells, we labeled dissociated cells with CFDA and cultured them in vitro. Wildtype neurospheres were enzymatically dissociated and subsequently labeled with $10 \mu \mathrm{M}$ CFDA as per the manufacturer's instructions. Virtually all cells (>98\%) of the dissociated neurospheres acquired CFDA and fluoresced immediately after labeling. Similar efficiencies were seen 3 hours after labeling. Dissociated cells labeled with CFDA were cultured in serum free medium supplemented with EGF, FGF-2 and heparin. After 7 days in culture, CFDA labeled cells produced neurospheres containing cells that remained labeled with CFDA. CFDA labeled cells were found primarily within the core of the neurospheres, with most peripheral cells showing no fluorescence (Figure 3A).

CFDA labeled cells were also plated on poly-L-lysine/laminin coated glass coverslips in reduced-growth factor medium and differentiated
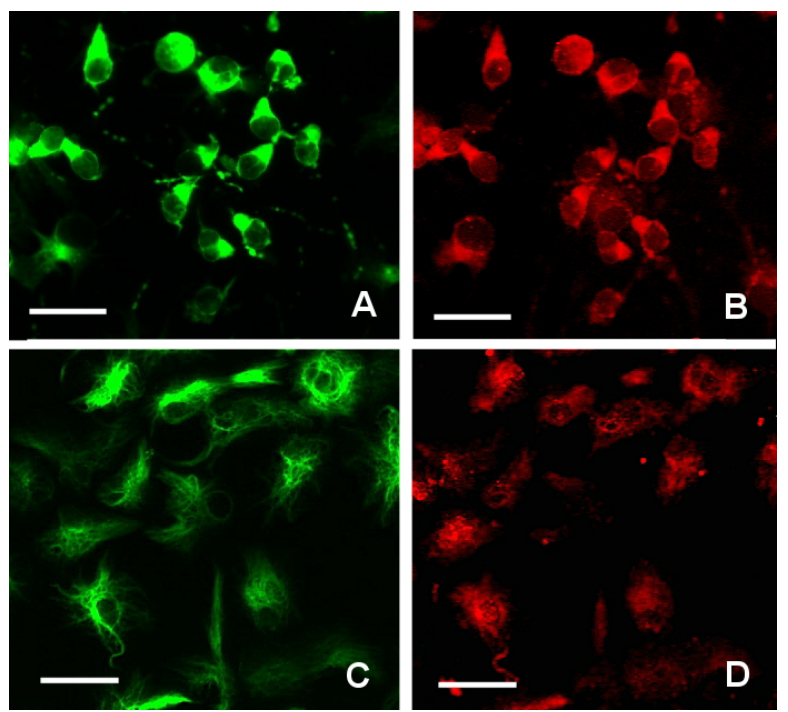

Figure 2: Expression of FMRP by neuronal and glial cells from wild type mice. A) Co-expression of a neuronal marker (BIII-tubulin) and (B) FMRP by neurosphere derived cells after 7 days in culture. Typical appearance of cells co-stained with (C) GFAP and (D) FMRP. Scale bar $=25 \mu \mathrm{m}$.
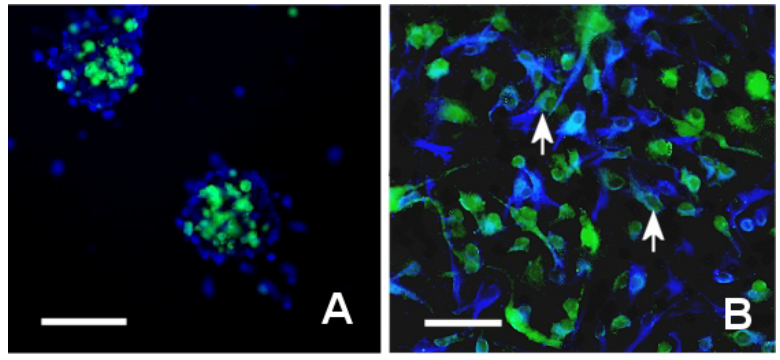

Figure 3: CFDA labelled cells. A) Neurosphere cores are heavily labelled with CFDA after 7 days in vitro. B) Differentiated cells contain the green CFDA label. In this field several cells co-express GFAP (arrows) with the CFDA marker. Scale bar $=100 \mu \mathrm{m}$ for $(A)$ and $50 \mu \mathrm{m}$ for $(B)$.

for 7 days in vitro. Differentiated cells retained the fluorescent label within the cytoplasm and processes.

However, the intensity of staining was much lower in differentiated cells than in neurospheres after 7 days in vitro. Although we did not fully characterize CFDA labeled cells in vitro, the cell survival and morphology appeared similar to unlabeled cells after differentiation. A high percentage of CFDA labeled cells also expressed GFAP, similar to non-labeled cells (Figure 3B).

\section{CFDA labeled transplants}

CFDA labeled cells were observed in the hippocampus and overlying cerebral cortex as early as 24 hours and as late as 2 weeks after transplantation. As previously reported [9], CFDA labeling produced high background fluorescence in the area of the transplant. This high background, combined with the density of cells within the transplant made accurate quantification of the number of surviving cells within the transplant difficult. We utilized an injection procedure whereby the cell suspension was injected in $1 \mu \mathrm{L}$ aliquots over approximately a 1.0 mm region (Figure $4 \mathrm{~A}, \mathrm{~B}, \mathrm{C}$ and $\mathrm{D}$ ). This method provided multiple transplant "hot-spots" and was successful to disperse the transplanted cells, but not sufficiently enough to quantify the number of labeled cells in the transplant. An obvious decrease in the number of cells, size of transplant and amount of background fluorescence was observed with increased time after transplantation.

To confirm that CFDA did not label the host tissue, dead CFDA labeled cells were transplanted to the brains of FMR1 knockout mice. One week after injection, high background fluorescence was observed similar to the treatment group. However, no individual CFDA labeled cells were observed, indicating that CFDA from dead cells did not label the host tissue.

FMRP expressing cells were found in the hippocampus and cortex in 4 of 5 animals 1 week after transplantation and in 3 of 5 animals after 2 weeks (Figure 4E).

One animal also showed FMRP expressing cells in the hippocampus and cortex 24 hours after transplantation (Figure 4F). No FMRP expressing cells were observed in any regions at three weeks posttransplantation ( $n=4$; not shown). Moderate FMRP expression was also detected in the 6 month old host brain one week after transplantation, suggesting that FMRP replacement is possible in both the young and old mouse brain.

The number of FMRP expressing cells varied widely between transplants. Overall, we noted significant levels of FMRP expression 
around the transplant in only a few mice. Numerous transplants showed very low levels, with only a small number (less than 5 per section) of cells expressing FMRP. In addition, other transplants showed no apparent FMRP expression. Not all sections from every transplant were processed for FMRP immunoreactivity; therefore, it is possible that additional FMRP expressing cells may have been found on sections processed for the other markers. Individual FMRP expressing cells were most easily detected when they were slightly peripheral to the central mass of transplanted cells. FMRP expression was confined to cells surrounding the transplant and was not evident in brain regions that did not receive a transplant. Since FMR1 knockout mice are characterized by complete absence of FMRP expression, we conclude that all FMRP expressing cells represent transplanted cells.

\section{Transplanted cells migrate into the host brain}

The majority of transplanted CFDA labeled cells remained within close proximity of the needle tract (Figure 5A). We noted groups of cells that migrated several hundred microns away from the needle tract in a medial/lateral direction. Many of these cells migrated in the white matter tracts, particularly the corpus callosum overlying the hippocampus. Since the needle insertion was oriented approximately perpendicular to the corpus callosum, the presence of cells in this region either medial or, most commonly lateral to the site of injection, indicated cell migration away from the injection site. We also observed some sections anterior and posterior to the injection site containing
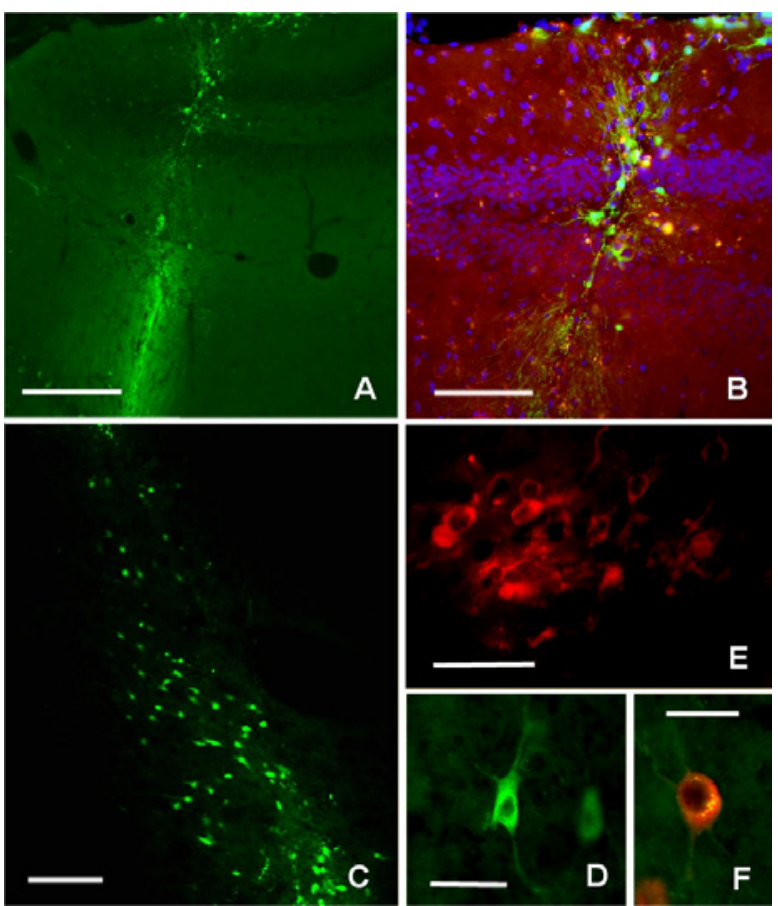

Figure 4: Transplants of neurosphere cell suspensions. A) Cell transplant located in the hippocampus is characterized by a linear tract of the the CFDA labeled cells ( 24 hours post-transplantation). Scale bar $=200 \mu \mathrm{m}$. B) Higher magnification of the transplant in (A) shows individual cells and processes within the dentate gyrus. Section is counterstained with DAPI to visualize the nuclei. Scale bar $=100 \mu \mathrm{m}$. C) Transplant located in the cerebral cortex shows numerous CFDA containing cells confined to the path of the needle tract. Scale bar $=100 \mu \mathrm{m}$. D) High magnification to illustrate the details of a CFDA labelled cell. Scale bar $=25 \mu \mathrm{m}$. E) Group of cells in a 7 day old transplant that express FMRP. Scale bar $=50 \mu \mathrm{m}$. F). Single cell stained for FMRP and containing the green CFDA label in the cytoplasm. Scale bar $=20 \mu \mathrm{m}$.
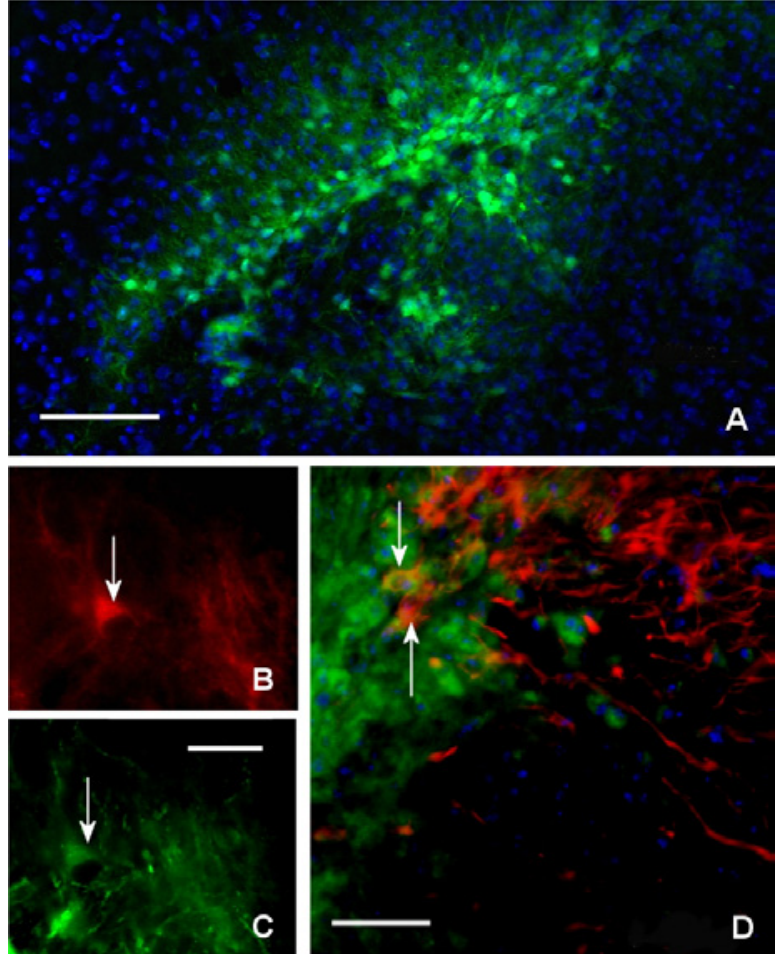

Figure 5: Differentiation of transplanted cells in vivo. A) Cluster of CFDA labeled cells with extensive processes that penetrate into the host neuropil. The linear direction of the needle within the cerebral cortex is evident. Scale bar $=$ $100 \mu \mathrm{m}$. B) Single cell expressing $\beta$ III tubulin is also labeled with the CFDA as shown in (C). Scale bar $=25 \mu \mathrm{m}$ for $(B)$ and (C). D) Immunofluorescent staining of CFDA cells with GFAP (arrows). Numerous processes within and around the transplant site are visible. Seven day old transplants. Scale bar $=50 \mu \mathrm{m}$.

transplanted cells exclusively within the corpus callosum. This suggests that cells had also migrated in an anterior-posterior direction within the white matter. Based on the number of sections obtained from each brain containing the transplant (CFDA-fluorescence), we estimated the transplants to have spanned an average of approximately 1.5 to $2.0 \mathrm{~mm}$ in an anterior-posterior plane after one week. The transplant size and the amount of CFDA fluorescence decreased noticeably with time after one week. Migration was also observed in the parenchyma of the cortex and hippocampus, albeit to a lesser extent than in the white matter. Transplanted cells appeared to remain within the region in which they were injected, as no CFDA labeled cells were identified in adjacent brain regions.

In many transplants we observed cells that extended CFDA-labeled processes into the surrounding tissue after 24 hours, one and two weeks. These cells tended to be slightly removed from the needle tract. In many transplants, we observed large bundles of long, thin processes, many extended in the same orientation. Generally, the cell bodies of these cells were not readily distinguishable. We also noted some cells with shorter, thicker processes, where the cell soma and processes were more uniformly labeled.

Some CFDA labeled cells also appeared to integrate into the cytoarchitecture of the host brain. These cells were most obvious in the hippocampus, where CFDA labeled cells in many transplants appeared to integrate into the granule and pyramidal cell layers of the hippocampus. Many of these cells also extended processes into 
the surrounding parenchyma. Cells were seen integrating into the hippocampus and extending processes to the surrounding tissue as early as 24 hours after transplantation but were most abundant one week after transplantation.

\section{Transplanted cells express glial and neuronal markers in vivo}

In order to further characterize the fate of transplanted neurosphere derived cells, we performed immunocytochemistry on transplanted brain sections using a variety of antibodies directed to specific cell fates. Some cells that retained the CFDA label expressed markers of a neuronal or a glial lineage. These results, however, were highly variable. Most CFDA labeled cells did not stain for any of the markers examined.

Occasional $\beta$ III tubulin expression was noted in CFDA labeled cells (Figure 5B and C). Some CFDA-labeled cells in the hippocampus/ cortex stained for gamma aminobutyric acid (GAD 65/67) (not shown), suggesting a GABA-ergic neuronal phenotype (not shown). GFAP expression appeared upregulated around the needle tract of some transplants after 7 days and 14 days. Some CFDA labeled cells in the hippocampus and cortex expressed GFAP when analyzed 7 days after transplantation (Figure 5D). No GFAP expression was observed at 24 hours in the vicinity of the transplant.

\section{Discussion}

Our results demonstrate the ability of wildtype neurospheres to produce FMRP expressing cells when transplanted to the brains of 3-6 week old FMRP knockout mice. FMRP expressing cells were observed 1, 7 and 14 days after transplantation. However, the number of FMRP expressing cells varied widely between individual transplants. Some transplants showed moderate FMRP expression around the injection site; however, many showed very few cells expressing FMRP. These findings suggest that FMRP expression in transplanted cells is present early after transplantation, and can persist for at least 2 weeks. We also detected FMRP expressing cells, in relatively high numbers, in a 6 month old mouse, one week after transplantation. This indicates that transplanted neurosphere-derived cells are capable of expressing FMRP in the adult mouse brain.

In vitro differentiation of neurosphere derived cells indicated the potential of these cells to form both neurons and glia. In order to characterize the cell types produced after transplantation, we stained brain sections with the same antibodies used on differentiated cells in culture. Most transplants showed varying numbers of cells labeled with CFDA, indicating the survival of transplanted cells. There was also an obvious decline in the number of CFDA labeled transplanted cells at two and three weeks post transplant as compared to one week. This may be due to increased cell death or a loss of the label with increased time after transplantation. However, although we noted robust staining of most antibodies in culture, very few cells expressed the majority of these markers after transplantation. We did note some cells expressing markers of neuronal or glial phenotypes. Many cells around the transplant also expressed nestin, a marker of immature cells. The low numbers of cells expressing the antigens tested, coupled with the high number of nestin positive cells around our transplants is consistent with the presence of undifferentiated cells. These findings are consistent with previous studies that have reported that a high number of cells remain undifferentiated weeks after transplantation [2,10-12]. Based on the small numbers of cells that expressed markers of differentiation, we can conclude that neurosphere derived cells have the potential to differentiate into neurons and glia after transplantation to the adolescent and adult brain. Further studies are needed to more closely examine the fate of these cells in vivo.

FMRP is a cytoplasmic protein and is not secreted from the cell. Therefore, in order to replace FMRP expression in the knockout mouse brain, FMRP-expressing cells must not only be present, but presumably must also integrate into the host circuitry to interact with host cells. Previous studies have shown that NSCs can produce functional neurons and integrate into the host circuitry after transplantation [13-15]. We observed cells one and two weeks post transplantation that extended processes to the surrounding tissue. Some cells also appeared to integrate into the host cytoarchitecture, particularly in the hippocampus. This suggests possible functional integration of the transplanted cells into the host circuitry.

We also observed migration of transplanted cells away from the site of injection. Many cells migrated within the white matter of the corpus callosum in both medial/lateral and anterior/posterior directions. Migration in the white matter was observed as early as 24 hours after transplantation and appeared to increase at later time points. Migration within the parenchyma did not appear to be dramatically altered between time points. Although we did observe some migration, the majority of labelled cells remained within the vicinity of the transplant. Fricker et al. [16] previously reported cells that divided more rapidly tended to travel longer distances after transplantation. Our in vitro studies showed fewer CFDA labelled cells in the periphery of the neurosphere, where proliferation is highest [17], as compared to the core. It is therefore possible that we were unable to detect cells that had migrated significant distances due to the loss of the CFDA label through increased proliferation.

The variability in the transplant outcomes is most likely explained by heterogeneity in our starting neurosphere population. It has been suggested that lineage-restricted progenitor cells may demonstrate increased survival, migration and differentiation, compared to true stem cells, when transplanted to the postnatal or adult brain $[1,10,18]$. Therefore, populations of neurosphere cultures with higher proportions of lineage-restricted progenitor cells might produce better grafts and perhaps in our case, more FMRP expressing cells than cultures in which cells are less differentiated.

Based on the high levels of expression of FMRP in the brains of postnatal wildtype mice, we had anticipated using FMRP expression as a marker for detecting transplanted cells in vivo. However, our results clearly indicated that not all CFDA labeled transplanted cells expressed FMRP in vivo. Although FMRP expression was high (approximately $60 \%$ ) in our differentiated cells in vitro, few transplanted cells expressed FMRP in vivo. There are a number of possible explanations for this finding. Several studies have demonstrated that patterns of gene expression in vitro can be altered when cells are transplanted in vivo $[19,20]$. These findings suggest that environmental cues in the host tissue differentially regulate gene expression in NSPCs in vivo. It is possible that we see similar downregulation in FMRP expression after transplantation into the FMR1 knockout brain, accounting for the low expression of FMRP in transplanted cells in vivo. In addition, FMRP expression is activity-dependent [21,22]; therefore, high levels of expression of FMRP in our transplanted cells may rely on transplanted cells to establish connections/contacts with other cells. Unknown environmental factors may also influence the expression 
Citation: Pacey LKK, Doering LC (2012) Expression of FMRP by Neurosphere Derived Cells Following Transplantation in the Fragile X Mouse Model. J Stem Cell Res Ther S12:001. doi:10.4172/2157-7633.S12-001

of FMRP in transplanted cells in the knockout brain. Gaining a better understanding of the mechanisms governing the expression of FMRP could aid in developing methods to increase FMRP expression in neurosphere-derived cells after transplantation.

A number of recent papers have illustrated defects in the differentiation of neural stem cells in the Fragile X mouse model $[23,24]$. In addition, FMRP has been linked with the control of proliferation and differentiation of adult neural/progenitor cells [25]. Specific examples of FMRP control include regulation of the transition from the radial glial cell to the intermediate progenitor [26] and the regulation of olfactory bulb neuron differentiation [27]. Hence, the global lack of FMRP in the knockout mouse may also contribute to the reduced FMRP expression in our transplanted cells.

In conclusion, we have demonstrated that neurosphere derived cells survive following transplantation to the hippocampus and cortex of the FMR1 knockout mouse. FMRP expressing cells were detected in many transplants up to two weeks after grafting. Transplants varied dramatically in cellular phenotype, possibly due to subtle differences in the culture conditions prior to transplantation, the inherent heterogeneity of the neurospheres or the global lack of FMRP that could influence the transplant microenvironment.

\section{Acknowledgements}

This research was supported by grants from the Natural Sciences and Engineering Research of Canada (NSERC) and the Fragile X Research Foundation of Canada.

\section{References}

1. Lepore AC, Neuhuber B, Connors TM, Han SS, Liu Y, et al. (2006) Long-term fate of neural precursor cells following transplantation into developing and adult CNS. Neuroscience 142: 287-304.

2. Shihabuddin LS, Horner PJ, Ray J, Gage FH (2000) Adult spinal cord stem cells generate neurons after transplantation in the adult dentate gyrus. J Neurosci 20: 8727-8735.

3. Eberhart DE, Malter HE, Feng Y, Warren ST (1996) The fragile X mental retardation protein is a ribonucleoprotein containing both nuclear localization and nuclear export signals. Hum Mol Genet 5: 1083-1091.

4. Bakker CE, de Diego OY, Bontekoe C, Raghoe P, Luteijn T, et al. (2000) Immunocytochemical and biochemical characterization of FMRP, FXR1P, and FXR2P in the mouse. Exp Cell Res 258: 162-170.

5. Devys D, Lutz Y, Rouyer N, Bellocq JP, Mandel JL (1993) The FMR-1 protein is cytoplasmic, most abundant in neurons and appears normal in carriers of a fragile X premutation. Nat Genet 4: 335-340

6. Bakker CE, Verheij C, Willemson R, van der Helm R, Oerlemans F, et al. (1994) FMR1 knockout mice: a model to study fragile $X$ mental retardation. Cell 78 : 23-33.

7. Pacey LKK, Stead S, Gleave JA, Tomczyk K, Doering LC (2006) Neural stem cell culture: neurosphere generation, microscopic analysis and cryopreservation. Nature Protocols DOI:10.1038/nprot(2006)215.

8. Pacey LKK, Doering LC (2007) Developmental expression of FMRP in the astrocyte lineage: Implications for Fragile X syndrome. Glia 55: 1601-1609.

9. Herrid M, Vignarajan S, Davey R, Dobrinski I, Hill JR (2006) Successful transplantation of bovine testicular cells to heterologous recipients. Reproduction 132: 617-624

10. Soares S, Sotelo C (2004) Adult neural stem cells from the mouse subventricula zone are limited in migratory ability compared to progenitor cells of similar origin. Neuroscience 128: 807-817.

11. Watson DJ, Walton RM, Magnitsky SG, Bulte JW, Poptani H, et al. (2006)

This article was originally published in a special issue, Neural Stem Cells handled by Editor(s). Dr. Thomas Lufkin, National University of Singapore, Singapore
Structure-specific patterns of neural stem cell engraftment after transplantation in the adult mouse brain. Hum Gene Ther 17: 693-704.

12. Burnstein RM, Foltynie T, He X, Menon DK, Svendsen CN, et al. (2004) Differentiation and migration of long term expanded human neural progenitors in a partial lesion model of Parkinson's disease. Int J Biochem 36: 702-713.

13. Klein C, Butt SJ, Machold RP, Johnson JE, Fishell G (2005) Cerebellum- and forebrain-derived stem cells possess intrinsic regional character. Development 132: 4497-4508.

14. Eriksson C, Bjorklund A, Wictorin K (2003) Neuronal differentiation following transplantation of expanded mouse neurosphere cultures derived from different embryonic forebrain regions. Exp Neurol 184: 615-635.

15. Ader M, Schachner M, Bartsch U (2004) Integration and differentiation of neura stem cells after transplantation into the dysmyelinated central nervous system of adult mice. Eur J Neurosci 20: 1205-1210.

16. Fricker RA, Carpenter MK, Winkler C, Greco C, Gates MA, et al. (1999) Sitespecific migration and neuronal differentiation of human neural progenitor cells after transplantation in the adult rat brain. J Neurosci 19: 5990-6005.

17. Campos LS (2004) Neurospheres: insights into neural stem cell biology, J Neurosci Res 78: 761-769.

18. Lepore AC, Han SS, Tyler-Polsz CJ, Cai J, Rao MS, et al. (2004) Differential fate of multipotent and lineage-restricted neural precursors following transplantation into the adult CNS. Neuron Glia Biol 1: 113-126.

19. Hofstetter CP, Holmstrom NA, Lilja JA, Schweinhardt P, Hao J, et al. (2005) Allodynia limits the usefulness of intraspinal neural stem cell grafts; directed differentiation improves outcome. Nat Neurosci 8: 346-353.

20. Jensen JB, Parmar M (2006) Strengths and limitations of the neurosphere culture system. Mol Neurobiology 34: 153-161.

21. Weiler IJ, Greenough WT (1999) Synaptic synthesis of the fragile X protein: possible involvement in synapse maturation and elimination. Am J Med Genet 83: $248-252$

22. Antar LN, Dictenberg JB, Plociniak M, Afroz R, Bassell GJ (2005) Localization of FMRP-associated mRNA granules and requirement of microtubules for activity-dependent trafficking in hippocampal neurons. Genes Brain Behav 4 350-359.

23. Castren M, Tervonen T, Karkkainen V, Heinonen S, Castren E, et al. (2005) Altered differentiation of neural stem cells in fragile $X$ syndrome. PNAS 102 17834-17839.

24. Castrén M (2006) Differentiation of neuronal cells in fragile $X$ syndrome. Cell Cycle 5: 1528-1530.

25. Luo Y, Shan G, Guo W, Smrt RD, Johnson EB, et al. (2010) Fragile X menta retardation protein regulates proliferation and differentiation of adult neural stem/progenitor cells. PLoS Genet 6: 1-15.

26. Saffary R, Xie Z (2011) FMRP regulates the transition from radial glial cells to intermediate progenitor cells during neocortical development. J. Neuroscience 31: $1427-1439$.

27. Scotto-Lomassese S, Nissant A, Mota T, Neant-Fery M, Oostra BA, et al (2011) Fragile $X$ mental retardation protein regulates new neuron differentiation in the adult olfactory bulb. Neuroscience 31: 2205-2215. 